\title{
Editorial-Autonomous Intelligent Systems
}

Jie Chen

Dear colleagues,

Welcome to the inaugural issue of Autonomous Intelligent Systems, a new open-source international journal launched to meet the needs of rapid publications and high visibility in autonomous systems, which introduces scholarly peer-reviewed works to everyone within the research community in a timely manner.

With the rapid development of artificial intelligence, "autonomous intelligent systems", as a relatively new interdisciplinary research field, has drawn more and more research attention over the past decades. Autonomy is usually characterized by the degree to which the system can achieve its objectives independently. An autonomous system should be able to run well in uncertain environments without human intervention, and handle problems caused by sensor failures, or poor communications, or physical damages. To achieve autonomy, the system should have a range of intelligent capabilities to complete complicated tasks and respond to unplanned and unforeseen scenarios.

There is a noticeable absence of journals devoted solely to publishing research and technological advances in autonomous and intelligent systems. With much effort to catch the opportunities, Springer Nature Publishing Group and Tongji University are very proud to launch a novel academic journal, Autonomous Intelligent Systems, a fully open access journal targeted to be one of the top international journals in the field of autonomous systems. The articles will be published online immediately upon acceptance to let researchers have free access to the most recent theoretical results and technological advances in the field of autonomous systems. In addition, the online submission and electronic peer review make the process of publishing articles simple and efficient, and there is no space constraint for the published paper.

Tongji University will sponsor Autonomous Intelligent Systems as an international and open platform for

Correspondence: chenjie206@tongji.edu.cn

Tongji University, 1239 Siping Road, Shanghai, China researchers and engineers of the autonomous intelligent system, which lies at the intersection of artificial intelligence, robotics, unmanned systems, multi-agent systems, complex networks, machine learning, cyberphysical systems, distributed decision making, humanrobot interaction, artificial intelligence ethics and laws, etc. It is worth mentioning that Tongji University established the Shanghai Research Institute for Autonomous Intelligent Systems in December 2018, for promoting and disseminating scientific discovery, knowledge, technology and applications in the fields related to autonomous intelligent systems. Affiliated with the institute, the journal will not only provide a venue for in-depth scientific exchanges but also seek to promote research collaborations all over the world.

Autonomous Intelligent Systems aims to publish topquality, peer-reviewed fundamental and applied research in the exciting and emerging fields of autonomous intelligent systems, which will cover following topics (but not limited to):

1) Intelligent robotics and developmental cognition;

2) Knowledge representation and natural language understanding or processing;

3) Multimedia systems and multi-sensor information fusion;

4) Analysis and control of multi-agent networks;

5) Machine learning and data-based decision making;

6) Swarm intelligence and distributed optimization;

7) Human-machine-environment interaction and network games;

8) Social networks and smart cities.

It will publish original research papers, surveys, letters, and book reviews to facilitate and speed up the dissemination of the new theories and innovation applications for autonomous intelligent systems. This journal has a distinguished editorial board to maintain its high scientific standards and broad international coverage. 
On behalf of the editorial board, we formally invite you to pay attention to and submit your best work to this journal, and would like to sincerely thank current and prospective authors, readers, editors, and reviewers for your strong support and contributions. For detailed information, please refer to the following journal website: https://www.springer.com/journal/43684

Wish Autonomous Intelligent Systems much success for years to come!

Jie Chen, Ph.D.

Editor-in-Chief, Autonomous Intelligent Systems IEEE Fellow, IFAC Fellow

Academician of Chinese Academy of Engineering, President and Professor of Tongji University

Shanghai, China

Published online: 17 August 2021

\section{Publisher's Note}

Springer Nature remains neutral with regard to jurisdictional claims in published maps and institutional affiliations. 